\title{
First measurement of the T-violating muon polarization in the decay $K^{+} \rightarrow \mu^{+} \nu \gamma$
}

\author{
V.V. Anisimovsky ${ }^{a *}$ A.N. Khotjantsev ${ }^{a}$, A.P. Ivashkin ${ }^{a}$, M. Abe $^{b}$, \\ M. Aliev ${ }^{a}$, M. Aokic, Y. Asano ${ }^{b}$, M. Blecher ${ }^{d}$, \\ P. Depommier ${ }^{e}$, M. Hasinoff $f$, K. Horie ${ }^{g}$, H.C. Huang ${ }^{h}$, \\ Y. Igarashi ${ }^{g}$, J. Imazato ${ }^{g}$, M.M. Khabibullin ${ }^{a}$, \\ Yu.G. Kudenko ${ }^{a}$, Y. Kuno ${ }^{c}$, A.S. Levchenko ${ }^{a}$, G.Y. Lim ${ }^{g}$, \\ J.A. Macdonald ${ }^{i}$, O.V. Mineev ${ }^{a}$, N. Okorokova ${ }^{a}$, \\ C. Rangacharyulu ${ }^{j}$, S. Shimizu ${ }^{c}$, Y.-M. Shin ${ }^{i}$, N.V. Yershov ${ }^{a}$, \\ T. Yokoi ${ }^{g}$ \\ (KEK E246 Collaboration) \\ ${ }^{a}$ Institute for Nuclear Research RAS, 117312 Moscow, Russia \\ ${ }^{b}$ Institute of Applied Physics, University of Tsukuba, Tsukuba, 305-0006, Japan \\ ${ }^{c}$ Osaka University, Osaka, 560-0043, Japan \\ ${ }^{d}$ Virginia Polytechnic Institute and State University, VA 24061-0435, USA \\ ${ }^{e}$ Université de Montréal, Montréal, H3C 3J77 Canada \\ ${ }^{f}$ University of British Columbia, Vancouver, V6T 2A3 Canada \\ ${ }^{g}$ High Energy Accelerator Research Organization (KEK), Tsukuba, 305-0801 Japan \\ ${ }^{h}$ Department of Physics, National Taiwan University, Taipei 106, Taiwan \\ ${ }^{i}$ TRIUMF, Vancouver, V6T $2 A 3$ Canada \\ ${ }^{j}$ University of Saskatchewan, Saskatoon, S7N 5E2 Canada
}

\begin{abstract}
We present the result of the first measurement of the T-violating transverse muon polarization $P_{T}$ in the decay $K^{+} \rightarrow \mu^{+} \nu \gamma$. This polarization is sensitive to new sources of $\mathrm{CP}$-violation in the Higgs sector. Using data accumulated in the period 1996-98 we have obtained $P_{T}=(-0.64 \pm 1.85$ (stat $) \pm 0.10$ (syst $\left.)\right) \times 10^{-2}$ which is consistent with no T-violation in this decay.
\end{abstract}

Key words: Kaon decays, muon polarization, T-violation

PACS: 11.30.Er; 12.60.-i; 13.20.Eb

\section{Introduction}

The decay $K^{+} \rightarrow \mu^{+} \nu \gamma\left(K_{\mu 2 \gamma}\right)$ is one of the kaon decays which could probe new physics beyond the Standard Model (SM). However, the decay is relatively poorly measured and there have

\footnotetext{
*Corresponding author. Email address: valera@al20.inr.troitsk.ru (V.V. Anisimovsky).
} 
been only a few experiments that determined the $K_{\mu 2 \gamma}$ branching ratio [1,2] and the structuredependent component [3]. Other interesting observables for this decay are the components of the muon polarization: $P_{T}$ which is the T-odd polarization component normal to the decay plane defined as $P_{T}=\vec{s}_{\mu} \cdot\left(\vec{p}_{\gamma} \times \vec{p}_{\mu}\right) /\left|\vec{p}_{\gamma} \times \vec{p}_{\mu}\right|$, and $P_{N}$-normal muon polarization (T-even) in the decay plane which is sensitive to the values and signs of the kaon vector $F_{V}$ and axial-vector $F_{A}$ form factors, as shown in 4. The contribution to $P_{T}$ from the Standard Model is known to be very small $\simeq 10^{-7}[5$. The T-even transverse polarization due to final state interactions (FSI) can mimic the T-odd effect at the $\leq 10^{-3}$ level [6, 7, 8, However, it can be reliably calculated. Therefore, any non-zero value of $P_{T}$ above the level of FSI or different from the FSI values, if the experimental sensitivity to the polarization is better than $10^{-3}$, would indicate new physics. Several non-SM models predict non-zero values for $P_{T}$ in $K_{\mu 2 \gamma}$ decay [9, 10,11] and from two of them (SUSY with R-parity and multi-Higgs models without natural flavor conservation) large values of $P_{T} \leq 10^{-2}$ are expected.

In this letter we present the first results on $P_{T}$ obtained in the analysis of the $K_{\mu 2 \gamma}$ data accumulated in the KEK E246 experiment for the 1996-98 data taking period.

\section{Experiment}

The measurement was performed using the detector constructed to search for T-violating muon polarization in the $K^{+} \rightarrow \pi^{0} \mu^{+} \nu\left(K_{\mu 3}\right)$ decay. The apparatus is shown in Fig. I and described

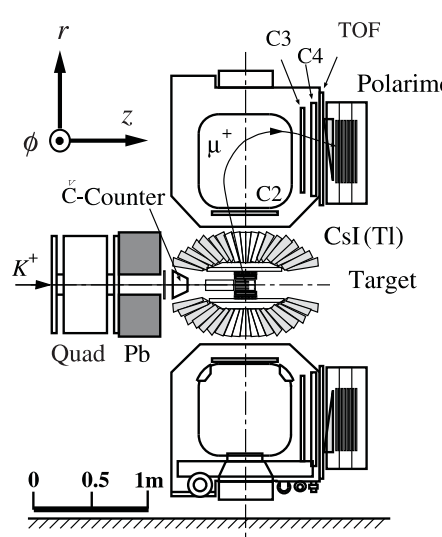

(a)

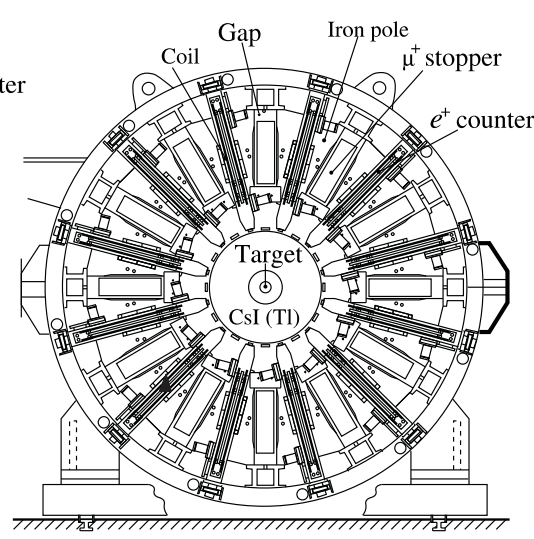

(b)

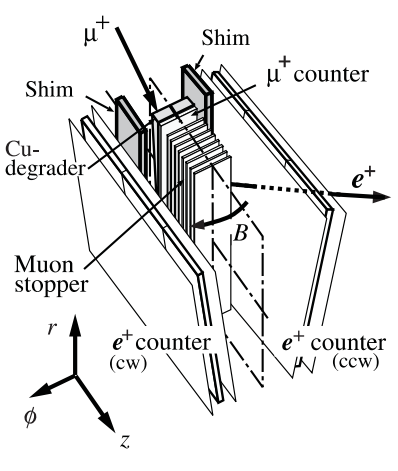

(c)

Figure 1: The layout of the KEK E246 detector:(a) side view, (b) end view and (c) one sector of the polarimeter.

in detail elsewhere 12 13 14,15. A separated $K^{+}$beam $(\pi / K \simeq 6)$ of $660 \mathrm{MeV} / c$ was used with a typical intensity of $3.0 \times 10^{5}$ kaons per 0.6 -s spill duration with a repetition of $3 \mathrm{~s}$. A Čerenkov counter with a multiplicity trigger distinguished $\mathrm{K}^{+} \mathrm{s}$ from $\pi^{+} \mathrm{s}$ with an efficiency of more than 99\%. Kaons then were slowed in a $\mathrm{Al}+\mathrm{BeO}$ degrader, and stopped in an active target made of 256 scintillating fibers located at the center of a 12-sector superconducting toroidal spectrometer. A charged particle $\left(\mu^{+}, e^{+}\right.$or $\left.\pi^{+}\right)$from the kaon decay hit one of the twelve fiducial counters surrounding the target and was bent through one of the spectrometer sector gaps and detected by the TOF and $\mu^{+}$counters at the entrance of the polarimeter. Tracking of charged particles was provided by the target fibers, a scintillation ring hodoscope surrounding the target and 
multiwire proportional chambers at the entrance $(\mathrm{C} 2)$ and exit $(\mathrm{C} 3$ and $\mathrm{C} 4)$ of each magnet sector gap. The momentum resolution of the whole tracking system was $\sigma_{p}=2.6 \mathrm{MeV} / c$ at $205 \mathrm{MeV} / c$.

The energies and angles of the photons were measured by the CsI photon calorimeter consisting of $768 \mathrm{CsI}(\mathrm{Tl})$ modules [15. The $\mathrm{CsI}(\mathrm{Tl})$ barrel has twelve holes for the charged particle entry to the toroidal spectrometer and covers a solid angle of about $0.75 \times 4 \pi$ sr. Energy resolution of $\sigma_{E} / E=2.7 \%$ at $200 \mathrm{MeV}$ and angular resolutions of $\sigma_{\theta}=2.3^{\circ}$ were obtained. Good time resolution of $3.5 \mathrm{~ns}$ (rms) at $100 \mathrm{MeV}$ allowed us to use the fast CsI signal at the trigger level and thereby effectively suppress accidentals in the calorimeter.

Muons entering the polarimeter (Fig. 1.) were degraded by an $\mathrm{Al}+\mathrm{Cu}$ block and stopped in a stack of pure $\mathrm{Al}$ plates. The stopper material $(\mathrm{Al})$ was chosen because there is no depolarization of muons stopped in aluminum. Positrons from $\mu^{+} \rightarrow e^{+} \nu \bar{\nu}$ decays of stopped muons were detected by 12 positron counters consisting of three layers of plastic scintillators which were located azimuthally between the muon stoppers. The time spectra of the positrons were recorded by multi-stop TDCs.

A three-level trigger for selection of events was used. At the first level, a signal from the kaon ring in the Cerenkov detector followed by a hit in at least one fiducial counter in coincidence with signals from the TOF and $\mu^{+}$counters of a corresponding magnet sector were required. The second level trigger required at least one hit in the $\mathrm{CsI}(\mathrm{Tl})$ calorimeter, and the third level trigger required the triple coincidence signal from the positron counter arrays adjacent to the corresponding magnet sector within $20 \mu$ s after the first level trigger.

The T-violating asymmetry was extracted using a double ratio as:

$$
A_{T}=\frac{1}{4}\left[\frac{\left(N_{c w} / N_{c c w}\right)_{f w d}}{\left(N_{c w} / N_{c c w}\right)_{b w d}}-1\right]
$$

Here, $N_{c w}$ and $N_{c c w}$ are the sums over all 12 sectors of counts of clockwise $(c w)$ and counterclockwise (ccw) emitted positrons. Indices fwd and bwd denote two classes of events: forward events $(f w d)$ when the angle between the photon and the beam direction (z-axis) was less than $70^{\circ}$ and backward events (bwd) when the angle between the photon and the beam direction was more than $110^{\circ}$. The signal values $N_{c w}$ and $N_{c c w}$ were extracted by integrating the positron time spectrum in the polarimeter after subtraction of the background. The signal integration region was chosen to be from 20 ns to $6 \mu$ s and the background fitting region for the background extraction was from 6.0 to $19.5 \mu \mathrm{s}$.

The sign of $A_{T}$ for events with forward-going photons is opposite to that for events with backward-going photons. This allows us to employ a double ratio method which is a powerful technique for reduction of most systematic errors. Moreover, considerable reduction of systematic effects was achieved from the azimuthal symmetry of the 12-gap detector and the use of the same positron detector as a $c w$ for one gap and a $c c w$ detector for the adjacent gap.

The value of $P_{T}$ is related to $A_{T}$ by

$$
P_{T}=\frac{A_{T}}{\alpha \times f \times(1-\beta)}
$$

where $\alpha$ is the analyzing power of the polarimeter, $f$ is an angular attenuation factor and $\beta$ is the overall fraction of all backgrounds. 


\section{Analysis}

The main problem of the $K_{\mu 2 \gamma}$ analysis is the rejection of the predominant $K_{\mu 3}$ background with only one photon $(1 \gamma)$ from the $\pi^{0}$ decay detected by the CsI calorimeter. The extraction of $K_{\mu 2 \gamma}$ events was performed in two stages.

The first stage was mostly identical to that of the main $\left(K_{\mu 3}\right)$ E246 analysis and was used to suppress $K_{\mu 2}, K_{\pi 2}, K_{e 3}$, and $K^{+} \rightarrow \pi^{+} \pi^{+} \pi^{-}, K^{+} \rightarrow \pi^{+} \pi^{0} \pi^{0}\left(K_{\pi 3}\right)$ background decays.

The $K_{\pi 2}$ and $K_{\mu 2}$ decays were rejected using a cut on the muon momentum $p_{\mu} \leq 190 \mathrm{MeV} / c$ selecting the $K_{\mu 2 \gamma}$ muons in the region of $100-190 \mathrm{MeV} / c$, as illustrated in Fig. 2. Most of the

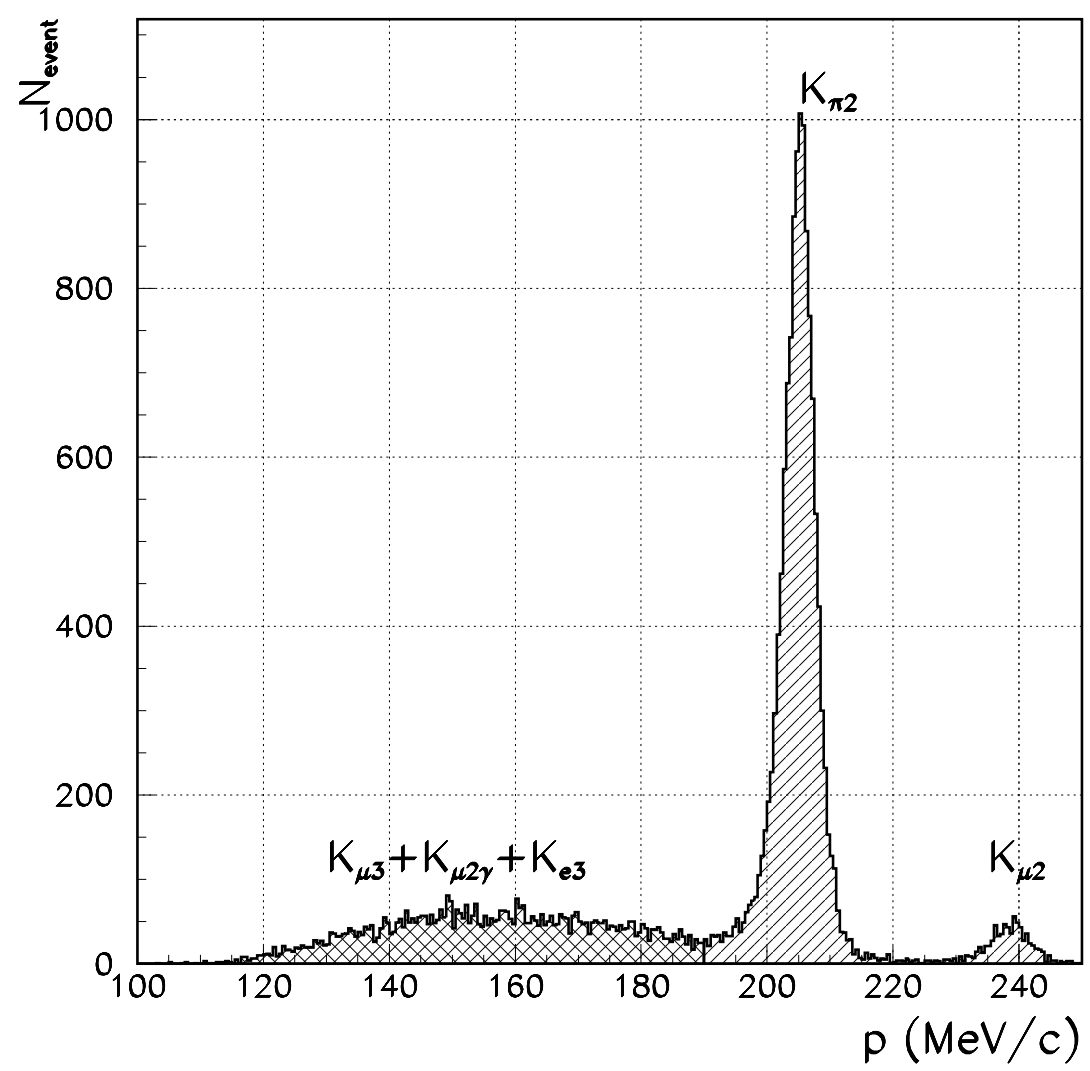

Figure 2: Momentum spectrum of charged particles for events with one photon detected by the CsI calorimeter. The double-hatched region shows the events with momentum less than 190 $\mathrm{MeV} / c$.

muons from $K_{\pi 2}$ events, in which $\pi^{+}$decays in-flight, were rejected by applying the tracking cut $\chi^{2}<10$. These events had kinks, i.e. the tracks did not point back to the kaon vertex. The $K_{\pi 3}$ backgrounds were negligible due to the combination of several suppressing factors: acceptance of the magnet spectrometer (the endpoints of charged particle momentum spectra for both decays are quite low: 125 and $133 \mathrm{MeV} / \mathrm{c}$, respectively), the low probability of detecting these decays as one-photon events and, finally, most of $\pi^{+} \mathrm{s}$ were stopped in the $\mathrm{Cu}$ degrader and did not reach 
the polarimeter. The $K_{e 3}$ events were removed using a time-of-flight method by imposing a geometric cut on the two-dimensional spectrum of a charged particle mass calculated from timeof-flight vs. the energy deposited in the TOF counter (Fig. B3). The background from accidental

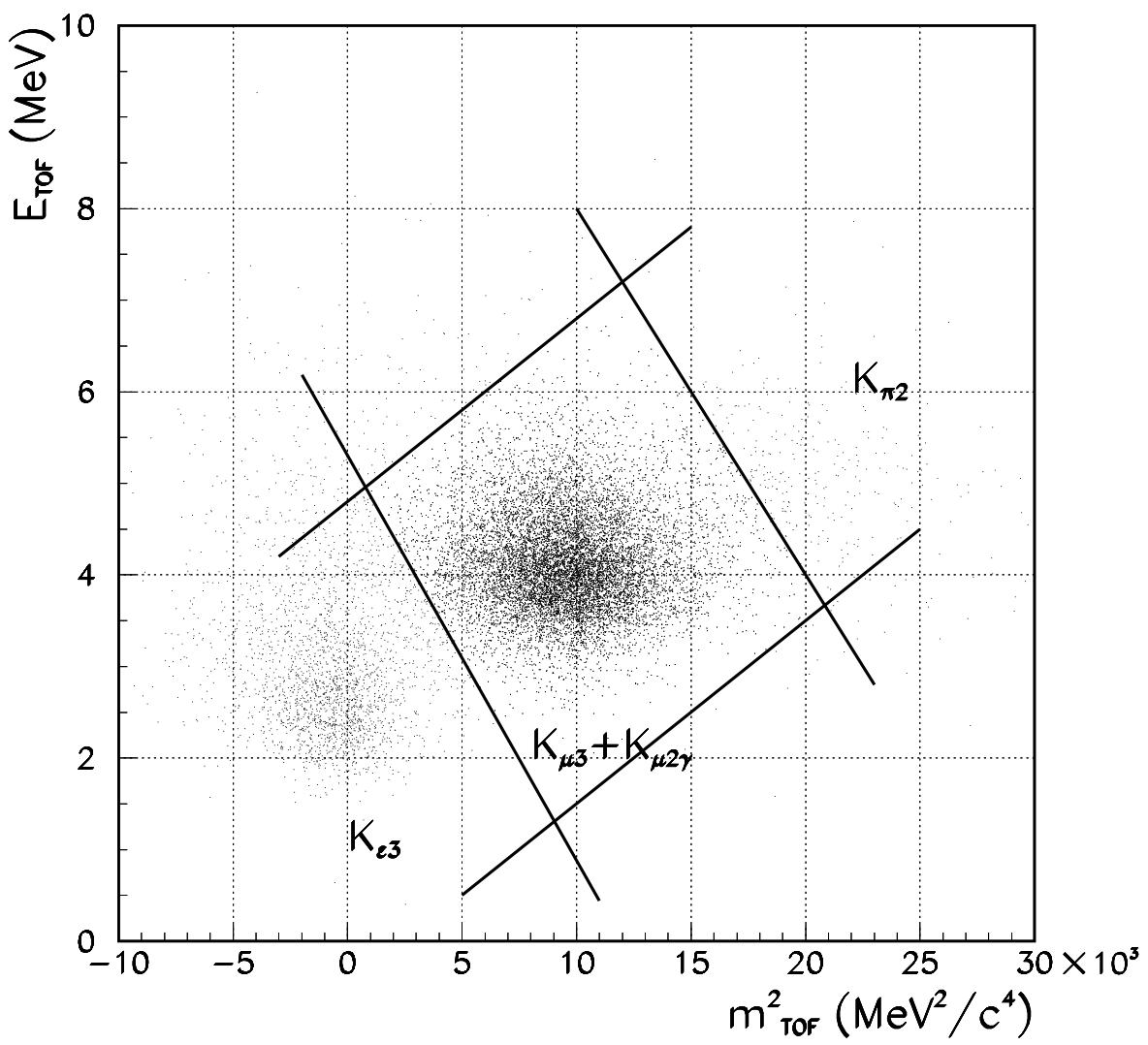

Figure 3: Rejection of $K_{e 3}$ using the time-of-flight technique. The "cloud" in the bottom-left corner corresponds to positrons, the events inside the rectangle are muons.

photons in the $\mathrm{CsI}(\mathrm{Tl})$ detector was suppressed using a photon energy threshold of $50 \mathrm{MeV}$ and by requiring a coincidence between a photon signal in the CsI and a signal in the fiducial and TOF counters from a charged particle within a window of \pm 15 ns. Background associated with the beam was suppressed by a veto counter system surrounding the beam region.

The second stage of the analysis was aimed at the extraction of $K_{\mu 2 \gamma}$ events and the rejection of $K_{\mu 3}$ decays. Due to the precise measurement of the muon momentum and the photon energy and direction, the kinematics of $K_{\mu 3}$ and $K_{\mu 2 \gamma}$ decays can be reconstructed completely [16]. The kinematic parameters such as missing mass squared $M_{m i s s}^{2}$, the angle between the muon and the photon, $\Theta_{\mu \gamma}$, and the neutrino momentum, $p_{\nu}$, can be efficiently used to suppress the $K_{\mu 3}$ background. The missing mass squared for $1 \gamma$ events is

$$
M_{m i s s}^{2}=E_{m i s s}^{2}-\vec{p}_{m i s s}^{2}=\left(m_{K}-E_{\mu}-E_{\gamma}\right)^{2}-\left(\vec{p}_{K}-\vec{p}_{\mu}-\vec{q}\right)^{2},
$$

where $\vec{q}$ is the photon momentum. For $K_{\mu 2 \gamma}$ events, the neutrino is the only missing particle, therefore, $\vec{p}_{\nu}=\vec{p}_{m i s s}, E_{\nu}=E_{m i s s}$ and $M_{m i s s}^{2}=E_{\nu}^{2}-\vec{p}_{\nu}^{2}=0$, while the $M_{m i s s}^{2}$ of $K_{\mu 3}$ events is 
distributed over a wide range with the maximum at about $2 \times 10^{4} \mathrm{MeV}^{2} / c^{4}$. The reconstructed neutrino momentum of $1 \gamma$ events from experimental and Monte Carlo (MC) data after imposing the cuts $-0.7 \times 10^{4} \mathrm{MeV}^{2} / c^{4}<M_{\text {miss }}^{2}<1.5 \times 10^{4} \mathrm{MeV}^{2} / c^{4}$ and $\Theta_{\mu \gamma} \leq 90^{\circ}$, and accepting events with $E_{\gamma}>50 \mathrm{MeV}$ is shown in Fig. प. The peak at $p_{\nu} \sim 220 \mathrm{MeV} / c$ in $\mathrm{MC}$ data corresponds

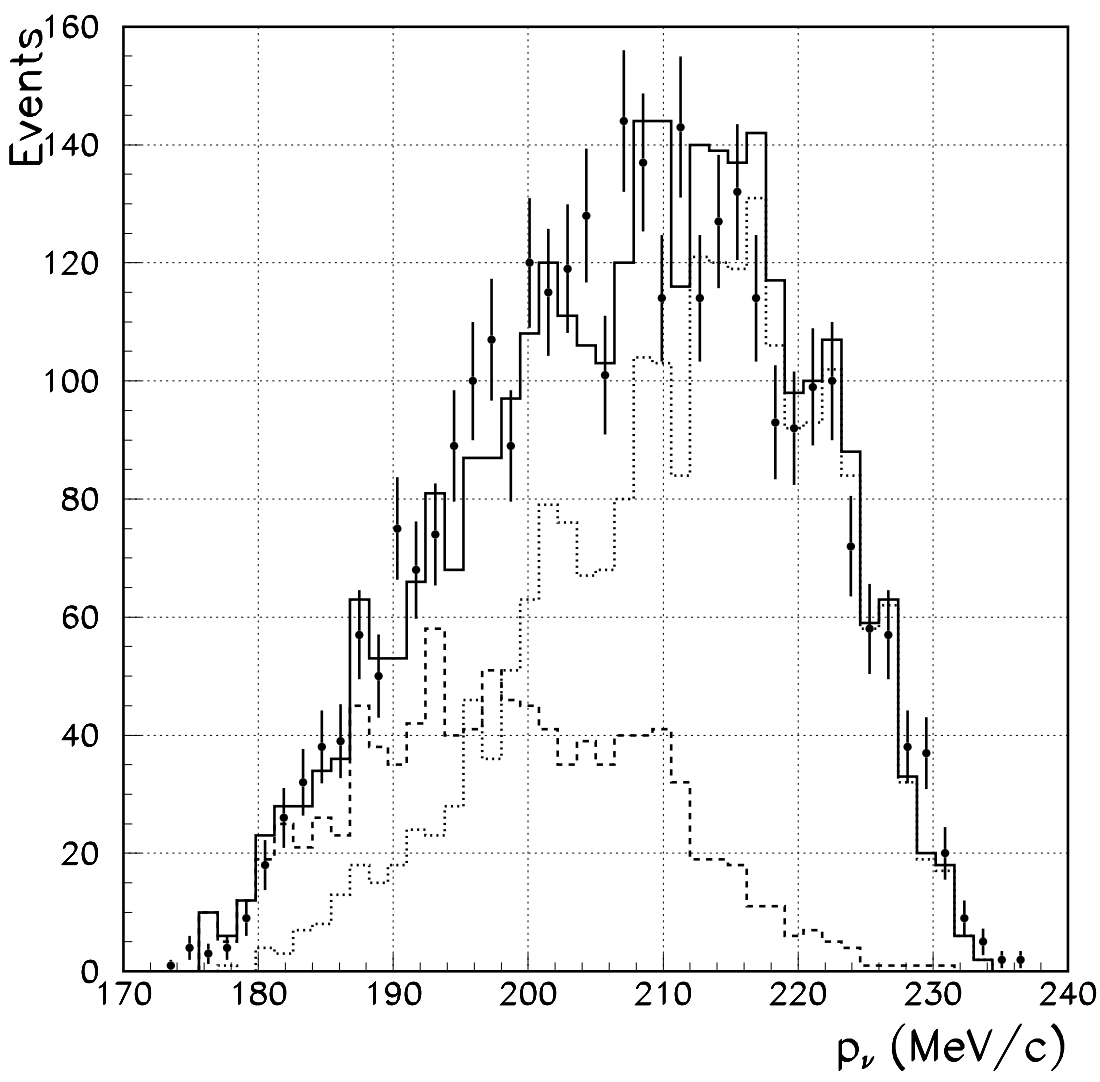

Figure 4: Momentum spectra of missing neutrino. The black dots with error bars show the experimental data. MC simulation: dotted line $-K_{\mu 2 \gamma}$, dashed line $-K_{\mu 3}$, solid line $-K_{\mu 2 \gamma}+K_{\mu 3}$. Cuts: $-0.7 \times 10^{4} \mathrm{MeV}^{2} / c^{4}<M_{\text {miss }}^{2}<1.5 \times 10^{4} \mathrm{MeV}^{2} / c^{4}, \Theta_{\mu \gamma}<90^{\circ}$.

to $K_{\mu 2 \gamma}$ events. In the region with $p_{\nu}>200 \mathrm{MeV} / c$, the contamination of the $K_{\mu 3}$ events is estimated to be $\simeq 17 \%$. Fig. [5] shows the experimental and MC spectra of neutrino missing mass after applying the cut $p_{\nu}>200 \mathrm{MeV} / c$.

After applying the cuts $p_{\nu}>200 \mathrm{MeV} / c,-0.7 \times 10^{4} \mathrm{MeV}^{2} / c^{4}<M_{m i s s}^{2}<1.5 \times 10^{4} \mathrm{MeV}^{2} / c^{4}$ and $\Theta_{\mu \gamma} \leq 90^{\circ}$, the accepted $K_{\mu 2 \gamma}$ events are concentrated in the Dalitz plot region, as shown in Fig. [6] where the inner bremsstrahlung (IB) component and interference of the IB and structuredependent with positive-helicity photon $\left(\mathrm{SD}^{+}\right)$term dominate.

A good test of the quality of detected $K_{\mu 2 \gamma}$ events is the value of the normal asymmetry, $A_{N}$. Its value is proportional to the T-even muon polarization, $P_{N}$, which is the in-plane component 


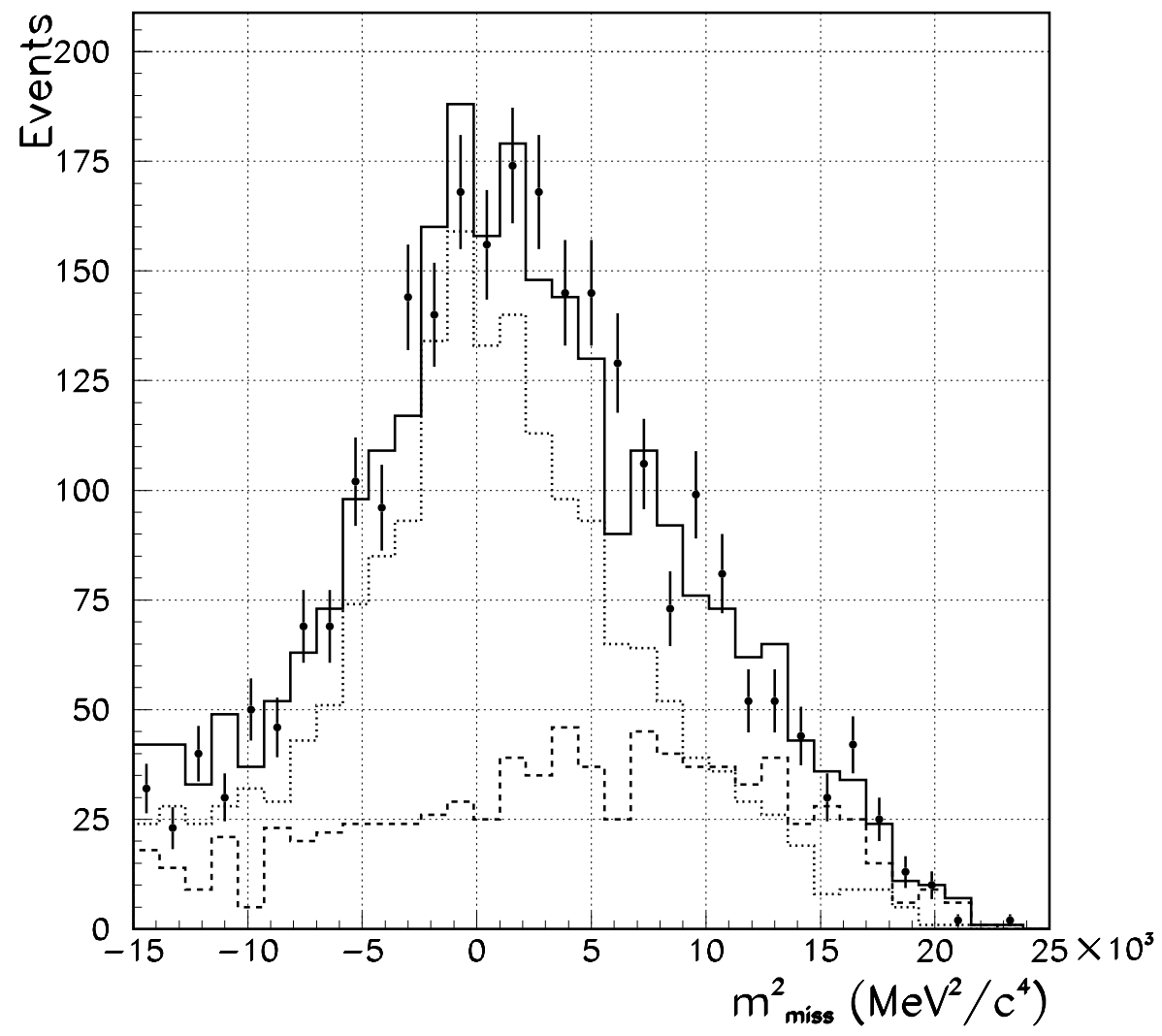

Figure 5: Missing mass spectra for the following cuts: $p_{\nu}>200 \mathrm{MeV} / \mathrm{c}, \Theta_{\mu \gamma}<90^{\circ}$. The black dots with error bars show the experimental data. MC simulation: dotted line $-K_{\mu 2 \gamma}$, dashed line $-K_{\mu 3}$, solid line $-K_{\mu 2 \gamma}+K_{\mu 3}$.

of the muon polarization normal to the muon momentum

$$
P_{N}=\frac{\vec{s}_{\mu} \cdot\left(\vec{p}_{\mu} \times\left(\vec{p}_{\gamma} \times \vec{p}_{\mu}\right)\right)}{\left|\vec{p}_{\mu} \times\left(\vec{p}_{\gamma} \times \vec{p}_{\mu}\right)\right|}
$$

$A_{N}$ can be measured if the accepted events are separated into two classes: events where the photon moves into the left hemisphere with respect to the median plane of the given magnet sector and events where the photon moves into the right hemisphere. The values of $A_{N}$ for these two classes should be the same but opposite in sign. For $K_{\mu 2 \gamma}$ the normal polarization has a positive sign in the region where the selected events are located [10, as seen from Fig. [6 and its average value is about 0.2 , while for $K_{\mu 3}$, the normal muon polarization calculated using the formulae of Ref. [17] has a negative sign over the whole Dalitz plot region, as shown in Fig. 7. From this measurement, the value of $A_{N}$ for the one-photon $K_{\mu 3}$ events is $A_{N}\left(K_{\mu 3}\right)=(-3.87 \pm$ $0.06) \times 10^{-2}$ and for selected $K_{\mu 2 \gamma}$ events with tighter cuts $-0.7 \times 10^{4} \mathrm{MeV}^{2} / c^{4}<M_{m i s s}^{2}<$ $1.0 \times 10^{4} \mathrm{MeV}^{2} / c^{4}, p_{\nu}>220 \mathrm{MeV} / c$ and $\Theta_{\mu \gamma} \leq 75^{\circ}$ the normal asymmetry is $A_{N}\left(K_{\mu 2 \gamma}\right)=$ $(3.59 \pm 0.56) \times 10^{-2}$. These values are both non-zero and have opposite signs consistent with the 


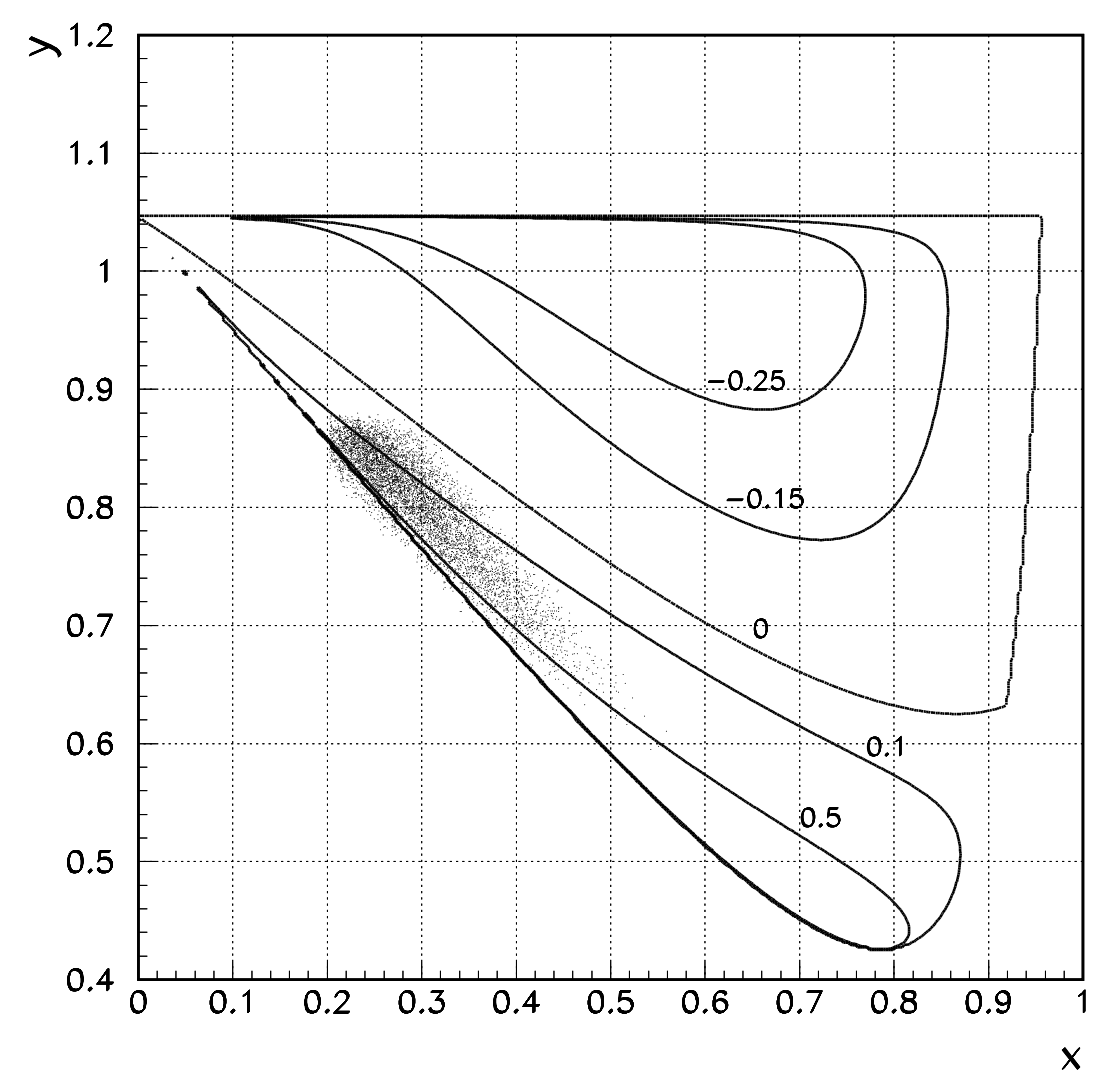

Figure 6: Contour lines of the normal polarization $P_{N}$ over the $K_{\mu 2 \gamma}$ Dalitz plot. The dots represent the experimental data after tight cuts: $-0.7 \times 10^{4} \mathrm{MeV}^{2} / c^{4}<M_{m i s s}^{2}<1.0 \times 10^{4} \mathrm{MeV}^{2} / c^{4}$, $p_{\nu}>220 \mathrm{MeV} / c, \Theta_{\mu \gamma} \leq 75^{\circ}, E_{\gamma}>50 \mathrm{MeV} ; x=\frac{2 E_{\gamma}}{m_{K}}$ and $y=\frac{2 E_{\mu}}{m_{K}}$.

expectation. That indicates a strong signature of the correct $K_{\mu 2 \gamma}$ selection and the suppression of one-photon $K_{\mu 3}$ events.

In Monte Carlo simulations the polarization value and corresponding asymmetry for $K_{\mu 2 \gamma}$ was obtained using the form factors from Chiral Perturbation Theory (ChPT) $F_{V}=-0.095$, $F_{A}=-0.043$ [18] and $f_{K}=159 \mathrm{MeV}$. After applying the same cuts to the MC data we obtained $A_{N}\left(K_{\mu 2 \gamma}\right)=(4.06 \pm 1.14) \times 10^{-2}$. These simulations were used to check the polarimeter sensitivity to muon polarization, i.e. the value of the analyzing power $\alpha$, and the geometric attenuation factor $f$. The $\alpha$ value obtained from $K_{\mu 2 \gamma}$ is in a good agreement with $\alpha=0.289 \pm$ 0.015 [19] extracted from $K_{\mu 3} \mathrm{MC}$ simulation. The attenuation factor $f=0.80 \pm 0.03$ was obtained.

From the experimental data collected during the 1996-98 beam cycles, a total number of $1.14 \times 10^{5} \mathrm{fwd}+$ bwd $K_{\mu 2 \gamma}$ events were extracted. The value of the transverse asymmetry measured for the collected events is $A_{T}=(-0.099 \pm 0.320($ stat $)) \times 10^{-2}$. The contamination of the beam accidental backgrounds in $K_{\mu 2 \gamma}$ events was estimated to be not higher than $8 \%$. The constant background in the polarimeter was $11-12 \%$. Both these backgrounds only dilute the 


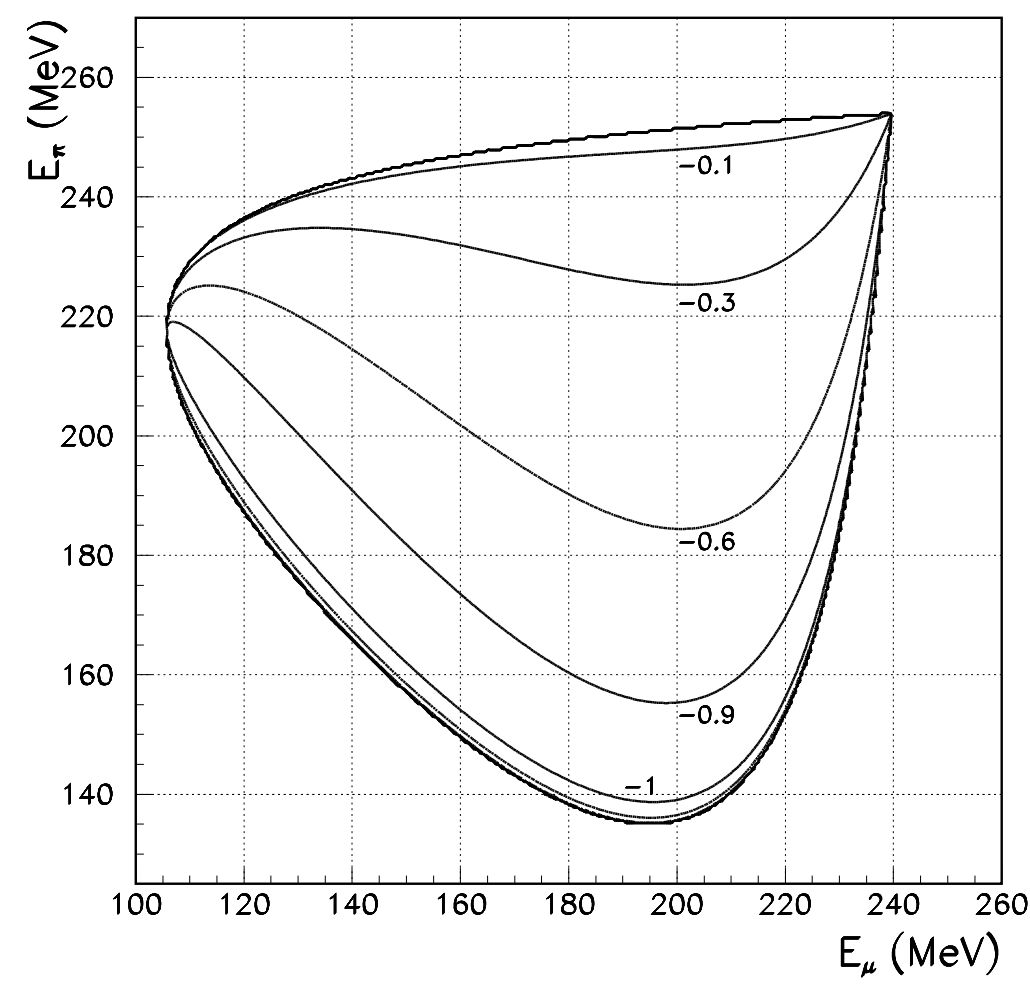

Figure 7: Contour lines of $P_{N}$ for the $K_{\mu 3}$ Dalitz plot.

sensitivity to $P_{T}$, but they do not produce any spurious T-violating asymmetry. Using MC data the background decay fractions were estimated to be $\beta_{b g r}^{K_{\mu 3}} \simeq 17 \%$ and $\beta_{b g r}^{K_{\pi 2}}<0.5 \%$. Thus, the total background contamination is $\beta \simeq 25 \%$ which is used in the denominator of Eq. (2).

Using the values of $A_{T}, \alpha, f$ and $\beta$ given above we obtained the transverse polarization $P_{T}=(-0.57 \pm 1.85($ stat $)) \times 10^{-2}$. The mean value of transverse polarization due to the final state interactions for the Dalitz plot region in which the selected $K_{\mu 2 \gamma}$ events reside is $P_{T}(\mathrm{FSI})=$ $0.7 \times 10^{-3}$ which is much less than the statistical error of $P_{T}$. The theoretical uncertainty of the $P_{T}(\mathrm{FSI})$ value is about $15 \%$ and is connected with the uncertainties of $F_{V}$ and $F_{A}$ values. For the final result we subtract $P_{T}(\mathrm{FSI})$ from the measured $P_{T}$ value which gives $P_{T}=(-0.64 \pm 1.85($ stat $)) \times 10^{-2}$ and as the $P_{T}$ (FSI) uncertainty is more than two orders of magnitude less than the statistical error we do not include that uncertainty in the $P_{T}$ error.

The main systematic error contributions to $P_{T}$ come from the presence of the two large components of the muon in-plane polarization, $P_{L}$ which is parallel to the muon momentum and $P_{N}$ $\left(P_{T} \ll P_{N, L} \leq 1\right)$. However, most of their contribution is canceled by the azimuthal symmetry of the detector as well as by the $f w d / b w d$ ratio. The largest instrumental systematic errors are due to the misalignment of the polarimeter, the asymmetry of the magnetic field distribution, and the asymmetric kaon stopping distribution. The $f w d / b w d$ ratio efficiently reduces these contributions. Additionally, a non-zero value of $P_{T}$ for the $K_{\mu 3}$ background would contribute to the systematic error of $P_{T}$ for $K_{\mu 2 \gamma}$, but that was measured in the main E246 analysis with much higher 
accuracy and is consistent with zero [19] $P_{T}\left(K_{\mu 3}\right)=(-1.12 \pm 2.17($ stat $) \pm 0.90($ syst $)) \times 10^{-3}$. All possible sources of systematic errors were considered in [20] for much higher statistics of $1 \gamma$ $K_{\mu 3}$ events as well as for $K_{\mu 2}$ and $K_{\pi 2}$ events. The total systematic error of $P_{T}$ for $K_{\mu 3}$ events was found to be $0.9 \times 10^{-3}$ [20], while for $K_{\mu 2 \gamma}$ we estimated the systematic error of $P_{T}$ to be less than $\delta P_{T}^{s y s}=1.0 \times 10^{-3}$. The absence of significant systematic errors (comparable to the statistical error) is confirmed by measuring the dependence of the transverse asymmetry $A_{T}$ on the magnet sector number as seen in Fig. 8.

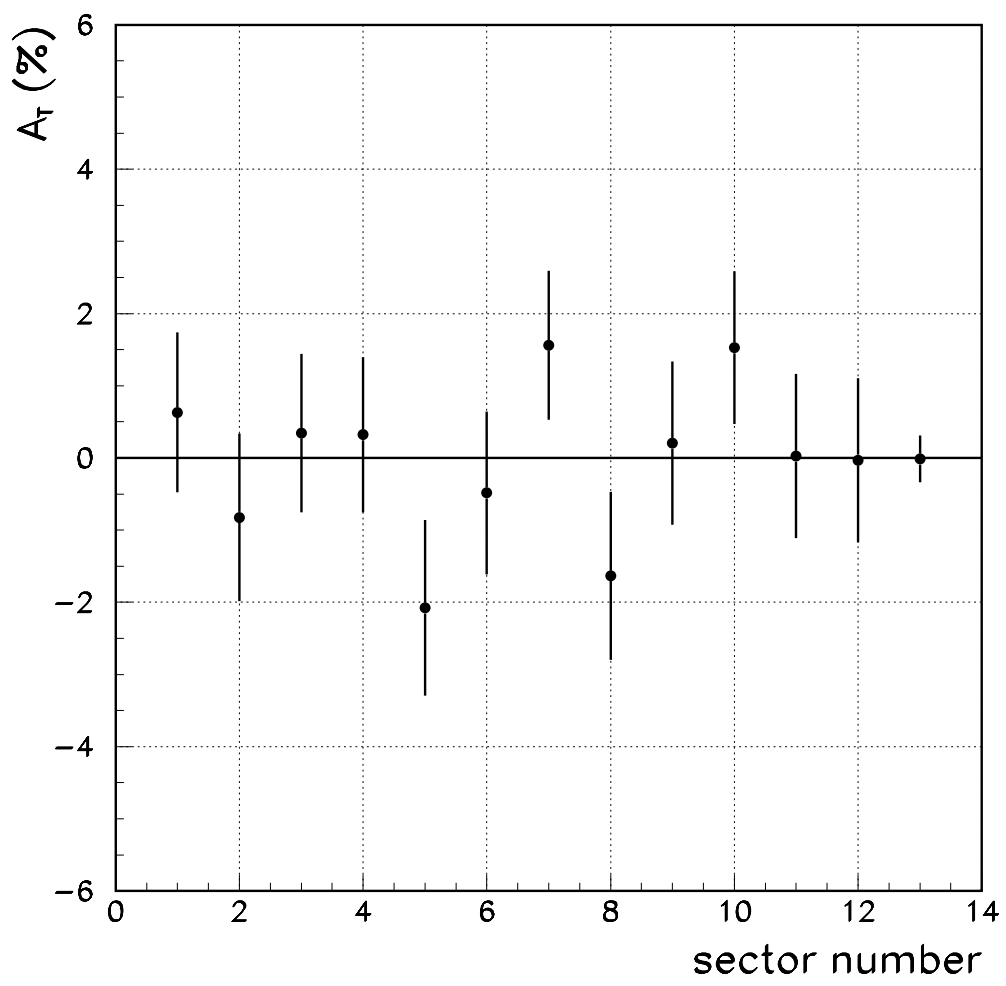

Figure 8: The dependence of the transverse asymmetry $\left(A_{T}\right)$ on the sector number. The rightmost point represents the sum of the asymmetries over all 12 sectors. The error bars show the statistical errors.

\section{Results}

We have performed the first measurement of the T-violating muon polarization in $K_{\mu 2 \gamma}$ decay based on about $1.14 \times 10^{5}$ good $f w d+$ bwd $K_{\mu 2 \gamma}$ events collected in 1996-98. These events are located in the Dalitz plot region where the IB and $\mathrm{INT}^{+}$components dominate. The value obtained for $P_{T}$ is $(-0.64 \pm 1.85$ (stat $) \pm 0.10($ syst $\left.)\right) \times 10^{-2}$ including the theoretical background from final state interactions. At the present level of experimental sensitivity this value is consistent with no T-violation in this decay. 


\section{Acknowledgments}

This work has been supported in Japan by a Grant-In-Aid from the Ministry of Education, Science, Sports and Culture, and by JSPS; in Russia by the Ministry of Science and Technology, and by the Russian Foundation for Basic Research; in Canada by NSERC and IPP, and by the TRIUMF infrastructure support provided under its NRC contribution; in the USA by the NSF. The authors gratefully acknowledge the support received from the INR and KEK staff.

\section{References}

[1] Y. Akiba et al., Phys. Rev. D32 (1985) 2911.

[2] V.S. Demidov et al., Sov. J. Nucl. Phys. 52 (1990) 1006.

[3] S.C. Adler et al., Phys. Rev. Lett. 85 (2000) 2256.

[4] F.L. Bezrukov, D.S. Gorbunov, and Yu.G. Kudenko, hep-ph/0302106.

[5] I. I. Bigi and A. I. Sanda, CP violation, Cambridge Monogr. Part. Phys. Nucl. Phys. Cosmol. 9, 1 (2000); E. Golowich and G. Valencia, Phys. Rev. D 40, 112 (1989).

[6] V. P. Efrosinin and Y. G. Kudenko, Phys. Atom. Nucl. 62 (1999) 987 [Yad. Fiz. 62 (1999) 1054].

[7] V.V. Braguta, A.E. Chalov, A.A. Likhoded, Phys. Rev. D66 (2002) 034012.

[8] R.N. Rogalev, Phys. Lett. B521 (2001) 243.

[9] M. Kobayashi, T.-T. Lin, and Y. Okada, Prog. Theor. Phys. 95 (1995) 361.

[10] C.H. Chen, C.Q. Geng, C.C. Lih, Phys. Rev. D56 (1997) 6856.

[11] G.-H. Wu, J. N. Ng, Phys. Rev. D55 (1997) 2806.

[12] M.P. Grigorev et.al., Instrum. Exp. Tech. 41 (1998) 803 [Prib. Tekh. Eksp. 41 (1998) 65].

[13] A.P. Ivashkin et al., Nucl. Instr. and Meth. A394 (1997) 321.

[14] M. Abe et al., Nucl. Instr. and Meth., to be published, hep-ex/0302001.

[15] A.D. Dementjev et al., Nucl. Instr. and Meth. A440 (2000) 151.

[16] Y. G. Kudenko, Phys. Atom. Nucl. 65 (2002) 244 [Yad. Fiz. 65 (2002) 269].

[17] N. Cabibbo and A. Maksymowicz, Phys. Lett. 9 (1964) 352; Errata ibid., 11 (1964) 360; Errata ibid.,14 (1965) 72.

[18] J. Bijnens, G. Ecker, J. Gasser, Nucl. Phys. B396 (1993) 81.

[19] M. Abe et al., hep-ex/0211049.

[20] M. Abe et al. [KEK-E246 Collaboration], Phys. Rev. Lett. 83 (1999) 4253. 